\title{
Chronic Glucocorticoid Therapy Amplifies Glomerular Injury in Rats with Renal Ablation
}

Diego L. Garcia, Helmut G. Rennke, Barry M. Brenner, and Sharon Anderson

With the technical assistance of J. L. Troy, L. E. Clarey, S. J. Downes, A. W. Nunn, S. L. Riley, K. J. Sandquist, and D. Sandstrom Laboratory of Kidney and Electrolyte Physiology, Departments of Medicine and Pathology, Brigham and Women's Hospital, Boston, Massachusetts 02115; and Harvard Medical School, Boston, Massachusetts 02115

\begin{abstract}
Functional and/or structural measurements were performed in eight groups of Munich-Wistar rats after five-sixths nephrectomy. Groups 1 and 5 received no therapy. Groups 2 and 6 received daily doses of methylprednisolone (MP). Groups 3 and 7 received MP plus the angiotensin I converting enzyme inhibitor (CEI), benzazepril. Groups 4 and 8 received CEI alone. Groups 1 through 4 underwent micropuncture study 2 wk after renal ablation. Untreated group 1 rats exhibited systemic hypertension and elevation of the single nephron glomerular filtration rate due to glomerular capillary hyperperfusion and hypertension. Administration of MP in group 2 resulted in comparable systemic hypertension, with further elevation of the single nephron glomerular filtration rate due to even higher values for glomerular perfusion and hydraulic pressure. Concurrent treatment with CEI in groups 3 and 4 controlled systemic and glomerular hypertension despite equivalent renal ablation and, in group 3, comparable doses of MP. Groups 5 through 8 were followed for 12 wk. Untreated group 1 rats demonstrated continued systemic hypertension, progressive proteinuria, and eventual glomerular sclerosis. Addition of MP in group 6 dramatically accelerated the development of proteinuria and glomerular sclerosis, while CEI (groups 7 and 8) afforded striking protection against disease progression. Thus, potent vasodilator glucocorticoids may amplify hemodynamically mediated glomerular injury, whereas control of systemic and glomerular hypertension prevents this undesirable consequence of chronic steroid therapy.
\end{abstract}

\section{Introduction}

Recent clinical studies have established that in patients with lupus nephritis, administration of cytotoxic drugs, often with low dose prednisone, is more likely than treatment with high dose prednisone alone to retard the progression of glomerular disease (1-4). This lesser therapeutic effectiveness of glucocor-

Portions of these studies were presented at the Annual Meeting of the American Federation for Clinical Research, Washington, D. C., in May, 1986, and at the 19th Annual Meeting of the American Society of Nephrology, Washington, D. C., in December, 1986, and were published in abstract form (1986. Clin. Res. 34:697; 1987. Kidney Int. 31:385).

Address reprint requests to Dr. Garcia, Renal Div., Brigham and Women's Hospital, 75 Francis St., Boston, MA 02115.

Received for publication 13 January 1987 and in revised form 1 May 1987.

J. Clin. Invest.

(C) The American Society for Clinical Investigation, Inc.

$0021-9738 / 87 / 09 / 0867 / 08 \quad \$ 2.00$

Volume 80, September 1987, 867-874 ticoids may be related to inadequate suppression of immunologic mechanisms of glomerular injury. Alternatively, we hypothesized that potent vasodilator steroids, which are known to increase glomerular perfusion and filtration (5-9), might contribute a hemodynamic burden to involved glomeruli, and thereby offset the potential benefit of the antiinflammatory action of these drugs.

Elevations in glomerular capillary pressures and flows have been associated with progression of renal injury in several experimental models, including surgical reduction of renal mass (10-12), streptozotocin-induced diabetes mellitus $(13,14)$, and mineralocorticoid-salt hypertension (15). In each of these models, increases in the single nephron glomerular filtration rate (SNGFR) ${ }^{1}$ result from elevations of the glomerular capillary plasma flow rate $\left(Q_{A}\right)$ and the mean glomerular transcapillary hydraulic pressure gradient $(\overline{\Delta \mathrm{P}})$. That these glomerular hemodynamic derangements contribute to structural injury is suggested by the findings that maneuvers which limit these increases in pressures and flows afford morphologic protection (10-16). Reduction of SNGFR, $Q_{A}$, and $\overrightarrow{\Delta P}$ with dietary protein restriction limits glomerular injury in each of these models (13, 15, 17-19). Alternatively, selective control of glomerular capillary hypertension with angiotensin I converting enzyme inhibitor (CEI) therapy limits glomerular injury in rats with renal ablation $(11,12)$ or diabetes (14) without affecting the supranormal plasma flow and filtration rates.

Conversely, maneuvers such as uninephrectomy $(20,21)$ or high protein feeding (17), which augment glomerular capillary pressures and flows, are associated with accelerated rates of progression. Chronic administration of pharmacologic doses of glucocorticoid hormones results in significant increases in glomerular filtration rate (GFR) in dogs (6), rats (5, $7)$, and humans $(8,9)$. In a micropuncture study of normal rats, Baylis and Brenner (5) found that administration of methylprednisolone (MP) resulted in single nephron hyperperfusion and hyperfiltration.

To examine the potential role of glucocorticoids to accelerate renal disease, we studied the hemodynamic and morphologic effects of chronic glucocorticoid administration in rats with renal ablation. This non-immunologic model was chosen, rather than an immunologically mediated form of renal disease, to avoid the uncertainties in evolution of progressive

1. Abbreviations used in this paper: $\overline{\mathrm{AP}}$, mean arterial blood pressure; CEI, converting enzyme inhibitor; Hct, hematocrit; $K_{f}$, glomerular capillary ultrafiltration coefficient; $\mathrm{MP}$, methylprednisolone; $\overline{\Delta \mathbf{P}}$, mean glomerular transcapillary hydraulic pressure gradient; $\overline{\mathbf{P}}_{\mathrm{GC}}$, mean glomerular capillary hydraulic pressure; $Q_{A}$, glomerular capillary plasma flow rate; $R_{A}$, afferent arteriolar resistance; $R_{E}$, efferent arteriolar resistance; SNGFR, single nephron glomerular filtration rate. 
injury that would result from variable modification of the inflammatory processes intrinsic to immunological injury.

\section{Methods}

Eight groups of male Munich-Wistar rats with initial weights of 220-250 g were used in these studies. All rats were subjected to fivesixth renal ablation by removal of the right kidney and infarction of approximately two-thirds of the left kidney by ligation of two or three branches of the left renal artery. All groups were fed standard rat chow (Ralston Purina Co., St. Louis, MO) containing 24\% protein by weight.

Groups 1 and 5 received no specific therapy. Groups 2 and 6 were treated with MP (Upjohn Co., Kalamazoo, MI), $15 \mathrm{mg} / \mathrm{kg}$ per d i.p., starting $5 \mathrm{~d}$ after ablation. This dose, which is one order of magnitude larger than that required for physiologic replacement in the rat (22), is comparable to pharmacologic doses ( $1 \mathrm{mg}$ prednisone $/ \mathrm{kg}$ ) used clinically. Groups 3 and 7 (MP/CEI) received MP in the same dose plus the CEI, benzazepril (Ciba-Geigy Pharmaceutical Co., Summit, NJ), at a dose of $100 \mathrm{mg} /$ liter in the drinking water, starting $5 \mathrm{~d}$ after ablation. Groups 4 and 8 received the CEI alone, at a dose of $50-100 \mathrm{mg} / \mathrm{liter}$, in the drinking water, starting $5 \mathrm{~d}$ after ablation. Groups 1 through 4 (each $n=7-8$ ) underwent micropuncture study 2 wk after ablation. Groups 5 through 8 (each $n=8-12$ ) were followed for 12 wk after ablation, at which time their remnant kidneys were perfusion fixed for evaluation of structural damage.

Systolic blood pressure was measured every $2 \mathrm{wk}$ in all rats in the conscious state by the tail-cuff method (23). 24-h urinary total protein excretion was measured in groups 5 through 8 at 3,6,9 and 12 wk after ablation.

Micropuncture studies. Groups 1 through 4 underwent micropuncture studies 2 wk after ablation. Rats were anesthetized with inactin $(100 \mathrm{mg} / \mathrm{kg}$ body weight i.p.) and placed on a temperature-regulated table. Immediately after the induction of anesthesia, the left femoral artery was catheterized with $\mathrm{PE}-50$ polyethylene tubing, followed by a baseline collection of $210 \mu \mathrm{l}$ of arterial blood. This arterial catheter was used for subsequent periodic blood sampling and estimation of mean arterial blood pressure $(\overline{\mathrm{AP}}) . \overline{\mathrm{AP}}$ was monitored with an electronic transducer (model P23Db; Statham Instruments Div., Gould Inc., Oxnard, CA) connected to a direct writing recorder (model 2200S; Statham Instruments Div., Gould Inc.). After tracheostomy, polyethylene catheters were also inserted into the jugular veins for infusions of inulin and plasma. Intravenous infusions of isoncotic rat plasma and $4 \%$ inulin solution in $0.9 \% \mathrm{NaCl}$ were started at rates of 6.0 and $1.2 \mathrm{ml} / \mathrm{h}$, respectively. The left kidney was then exposed by a subcostal incision, suspended on a Lucite holder, and its surface illuminated with a fiberoptic light source and bathed with isotonic $\mathrm{NaCl}$. The left ureter was catheterized with PE-10 tubing.

To compensate for the loss of plasma associated with anesthesia and surgery (24), the following protocol for maintaining the euvolemic state was used. After insertion of the jugular catheters, isoncotic rat plasma was infused for $20-30 \mathrm{~min}$ in a total amount equal to $1 \%$ of body weight, followed by a reduction in infusion rate to $0.4 \mathrm{ml} / \mathrm{h}$ for the remainder of the experiment to maintain the baseline hematocrit (Hct) value obtained immediately after induction of anesthesia. After a 60-min equilibration period, two 20-min timed urine collections were made. 140- $\mu$ l samples of femoral arterial blood were collected midway through each clearance period.

Micropuncture measurements were carried out as follows. Exactly timed (1-1.5 min) samples of tubule fluid were collected from surface proximal convolutions for determination of flow rate and inulin concentration, and calculation of SNGFR. Samples of efferent arteriolar blood were aspirated for determination of protein concentration. Coincident with these sample collections, $140 \mu \mathrm{l}$ of femoral arterial blood was obtained in each period for determination of Het and plasma concentrations of protein and inulin, and 15-20 min urine collections were obtained for determination of flow rate and inulin concentration. Time-averaged hydraulic pressures were measured in surface glomerular capillaries, proximal tubules, and efferent arterioles with a continuous recording, servo-null micropipette transducer system (model 3; Instrumentation for Physiology and Medicine, San Diego, CA). Hydraulic output from the servo system was coupled electronically to a second channel of the recorder by means of a pressure transducer. Colloid osmotic pressure of plasma entering and leaving glomerular capillaries was estimated from values for protein concentration in femoral arterial and surface efferent arteriolar plasma samples, using the equation derived by Deen et al. (25). Values for protein concentration, and thus colloid osmotic pressure, for femoral arterial plasma are taken as representative of values for these parameters for the afferent end of the glomerular capillary network. These estimates of preglomerular and postglomerular plasma protein concentration permit calculation of single nephron filtration fraction, glomerular capillary ultrafiltration coefficient $\left(K_{f}\right), Q_{A}$, glomerular and postglomerular blood flow rates, and single afferent and efferent arteriolar resistances $\left(R_{A}\right.$ and $R_{E}$, respectively), using equations described previously (25).

Morphology. Rats in groups 5 through 8 followed for 12 wk after ablation were prepared for morphologic examination. Kidneys were fixed by perfusion at the measured arterial pressure with $1.25 \%$ glutaraldehyde in $0.1 \mathrm{M}$ cacodylate buffer ( $\mathrm{pH}$ 7.4). After perfusion fixation, one or two 3-4-mm-thick coronal sections through the mid portion of the remnant kidney were postfixed in $4 \mathrm{~g} / 100 \mathrm{ml}$ buffered formaldehyde solution and processed for light microscopy through paraffin embedding. Sections $3 \mu \mathrm{m}$ in thickness were stained with hematoxylin/eosin and by the periodic acid-Schiff technique. The frequency of focal and segmental sclerosis was determined by examining all glomerular profiles (average, 218) contained in one coronal section from each animal. Segmental lesions were specifically defined as area of the tuft showing collapse of the glomerular capillaries, often accompanied by hyaline deposition and/or adhesion of the tuft to Bowman's capsule. For each animal, the number of glomeruli with segmental lesions was expressed as a percentage of the total number of glomerli counted. Other glomerular changes, such as expansion of the mesangial areas and abnormalities of arteries and arterioles, were assessed nonquantitatively by light microscopy. Small, randomly selected fragments of cortex were also processed by osmium postfixation and epoxy-resin embedding. 1- $\mu \mathrm{m}$-thick epoxy-resin sections were stained with $1 \%$ toluidine blue in $1 \%$ aqueous borax and examined by light microscopy for further delineation of glomerular lesions.

Analytical. The volume of fluid collected from individual proximal tubules was estimated from the length of the fluid column in a constant bore capillary tube of known internal diameter. The concentration of inulin in tubule fluid was measured, usually in duplicate, by the microfluorescence method of Vurek and Pegram (26). Inulin concentrations in plasma and urine were determined by the macro-anthrone method of Führ et al. (27). Protein concentrations in efferent arteriolar and femoral arterial blood plasma were determined, usually in duplicate, using a fluorometric method developed by Viets et al. (28). Urinary protein concentration was measured by precipitation with $3 \%$ sulfosalicylic acid (29).

Statistical. Statistical analysis was performed by one-way analysis of variance followed by computation of modified $t$ values and multiple pairwise comparisons according to the method of Bonferroni (30). Statistical significance was defined as $P<0.05$.

\section{Results}

General. MP-treated rats tended to lose more body weight than did untreated rats during the first 2-4 wk after renal ablation. Thereafter, all groups gained weight at comparable rates. Food and water intake were measured at random time points and were comparable among groups. Random blood glucose levels in MP-treated rats were never above the normal range, excluding the possibility of steroid-induced hyperglycemia. 
Systemic blood pressure. Untreated rats subjected to fivesixths nephrectomy developed systemic hypertension within 2 wk of renal ablation. As demonstrated in Fig. 1, systolic blood pressure in group 5 rats averaged $160 \pm 9 \mathrm{mmHg}$ (SEM) by 2 wk, and were maintained at values equal to or exceeding this level throughout the observation period. Hypertension of similar magnitude was observed in rats given MP. Despite equally extensive renal ablation, the development of systemic hypertension was largely prevented in rats treated with CEI alone or with MP/CEI (both $P<0.005$ vs. group 5).

Micropuncture studies. Table I summarizes the mean values for body weight, Hct, whole kidney GFR, AP, SNGFR, and the pressures, flows, and resistances governing glomerular ultrafiltration in groups 1 through 4 studied 2 wk after ablation. There were no significant differences in body weight or Hct among the four groups. Values for $\overline{\mathrm{AP}}$ were comparably elevated in untreated and MP-treated rats, whereas systemic hypertension was absent in both groups receiving CEI (both $P$ $<0.05$ vs. group 1). Groups 2 through 4 exhibited numerically higher values for whole remnant kidney GFR compared with untreated group 1 rats.

Single nephron hyperfiltration was apparent in all groups, with values considerably higher than those seen in normal rats (14). In the untreated group 1 rats, single nephron hyperfiltration resulted from elevations of $Q_{A}$, which averaged $219 \pm 18$ $\mathrm{nl} / \mathrm{min}$, and $\overline{\Delta \mathrm{P}}(53 \pm 2 \mathrm{mmHg})$. Since values for proximal tubule hydraulic pressure were equivalent in all groups, alterations in $\overline{\Delta \mathrm{P}}$ reflected differences in values for the mean glomerular capillary hydraulic pressure $\left(\bar{P}_{\mathrm{GC}}\right)$. Administration of benzazepril (group 4) resulted in slightly but not significantly higher values for both $Q_{A}$ and SNGFR as compared with the untreated group 1 rats. Converting enzyme inhibition with this agent, as has been shown using the CEI enalapril in rats with renal ablation $(11,12)$, resulted in control of glomerular capillary hypertension, so that values for $\overline{\mathrm{AP}}(35 \pm 2 \mathrm{mmHg})$ were maintained at near normal levels $(P<0.001$ vs. group 1$)$.

SBP $(\mathrm{mmHg})$

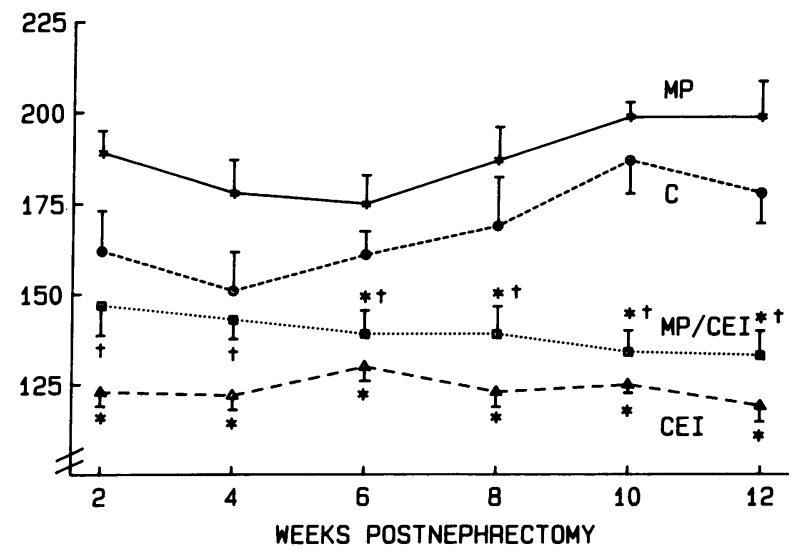

Figure 1. Systolic blood pressure (SBP). SBP measured by the tail cuff method in conscious rats followed for $12 \mathrm{wk}$ after five-sixths nephrectomy. Untreated (group 5, C) and MP-treated (group 6, MP) rats exhibited comparable sustained systemic hypertension, while addition of CEI to MP-treated rats (group 7, MP/CEI) or CEI alone (group 8, CEI) maintained SBP at normal levels. Values are means \pm SEM. $* P<0.05$ vs. $C,+P<0.05$ vs. MP at the same time point.
Values for $\mathrm{K}_{\boldsymbol{f}}$ were preserved at normal levels with CEI ( $P$ $<0.001$ vs. group 1). Values for efferent arteriolar hydraulic pressure, afferent arteriolar protein concentration, and colloid osmotic pressure were comparable in all groups. Values for efferent arteriolar protein concentration and colloid osmotic pressure in group 4 were slightly lower than in group 2 rats.

Administration of MP to group 2 rats produced important alterations in the hemodynamic determinants of glomerular ultrafiltration. SNGFR values were higher than in group 1 due to even further elevations of $Q_{A}(311 \pm 18 \mathrm{nl} / \mathrm{min})$ and $\overline{\Delta P}$ $(70 \pm 2 \mathrm{mmHg}$ ) (Table I). Enhanced glomerular perfusion resulted from a further reduction in total renal resistance, with a proportionately greater fall in afferent than efferent resistance. Transmission of systemic hypertension to the glomerular capillary network was thereby enhanced, resulting in extreme glomerular capillary hypertension and elevation of $\overline{\Delta \mathrm{P}}$. As in the untreated group 1 rats, glomerular capillary hypertension in the MP-treated group was associated with depressed values for $K_{f}$ compared with values seen in normal rats (14).

In the group 3 rats, concurrent administration of CEI to MP-treated rats resulted in control of systemic and glomerular capillary hypertension, with values for $\overline{\Delta \mathrm{P}}$ averaging only $39 \pm 1 \mathrm{mmHg}(P<0.001$ vs. groups 1 and 2$)$. Values for $\mathbf{R}_{\mathrm{A}}$ were reduced to levels comparable with those in MP-treated rats. $R_{E}$ was reduced as well, thereby preventing the rise in glomerular capillary hydraulic pressure that would otherwise occur. Control of glomerular hypertension in the MP/CEI rats (group 3) was accompanied by preservation of $K_{f}$ at normal levels $(0.100 \pm 0.011 \mathrm{nl} /(\mathrm{s} \cdot \mathrm{mmHg})(P<0.001$ vs. groups 1 and 2).

Proteinuria. Exposure to sustained systemic and glomerular hypertension in the untreated group 5 rats was associated with increasing levels of proteinuria throughout the 12 -wk observation period (Fig. 2). The markedly enhanced glomerular capillary hyperperfusion and hypertension resulting from MP administration were associated with acceleration of proteinuria, so that values in group 6 rats reached twice those in the untreated group 5 rats after $12 \mathrm{wk}(P<0.05)$. Control of systemic and glomerular capillary hypertension with CEI in groups 7 and 8 maintained protein excretion values at nearnormal levels, with virtually no progression over the $12 \mathrm{wk}$ of study (both $P<0.05$ vs. groups 5 and 6 ).

Structural alterations. At the end of the 12-wk observation period, untreated group 5 rats showed prominent and widespread glomerular alterations characterized by focal and segmental collapse of capillaries, hyaline deposition, and adhesion of the glomerular tuft to Bowman's capsule (Fig. $3 a$ ). These areas of collapse often contained vacuolated cells surrounded by basement membrane and matrix material. Focal and segmental obsolescence of the glomerular capillary tuft as described above was present in $18.5 \pm 2 \%$ of glomeruli in the untreated group 5 rats (Fig. 4). In addition, occasional glomeruli in untreated animals showed microaneurysm formation identical in appearance to the lesions described previously in mineralocorticoid-salt hypertension (15). Epithelial cell abnormalities with increased numbers of lysosomes (reabsorption droplets) and cytoplasmic blebs were often observed. Occasional areas of tubule atrophy, interstitial fibrosis and mild chronic inflammation, and cast formation in distal tubules and ascending thick segments of the loop of Henle were observed in association with the glomerular abnormalities. Arter- 
Table I. Summary of Renal Cortical Microcirculation Studies

\begin{tabular}{|c|c|c|c|c|c|c|c|c|c|}
\hline Group & $\begin{array}{l}\text { Body } \\
\text { weight }\end{array}$ & Het & $\overline{\mathbf{A P}}$ & GFR & SNGFR & SNFF & $Q_{A}$ & $\overline{\mathbf{P}}_{\mathbf{G C}}$ & $\mathbf{P}_{\mathbf{T}}$ \\
\hline & $g$ & $\mathrm{vol} / 100 \mathrm{ml}$ & $\mathrm{mmHg}$ & $\mathrm{ml} / \mathrm{min}$ & $n l / m i n$ & & $n l / m i n$ & $m m H g$ & $m m H g$ \\
\hline $1 \mathrm{C}(n=8)$ & $245 \pm 5$ & $45 \pm 2$ & $137 \pm 6$ & $0.51 \pm 0.05$ & $71 \pm 5$ & $0.33 \pm 0.01$ & $219 \pm 18$ & $69 \pm 2$ & $16 \pm 1$ \\
\hline $2 \mathrm{MP}(n=7)$ & $232 \pm 7$ & $47 \pm 1$ & $147 \pm 7$ & $0.72 \pm 0.08$ & $100 \pm 3$ & $0.33 \pm 0.02$ & $311 \pm 18$ & $85 \pm 2$ & $15 \pm 1$ \\
\hline $3 \mathrm{MP} / \mathrm{CEI}(n=8)$ & $230 \pm 5$ & $46 \pm 1$ & $109 \pm 4$ & $0.62 \pm 0.07$ & $83 \pm 5$ & $0.28 \pm 0.02$ & $305 \pm 27$ & $52 \pm 1$ & $13 \pm 0.4$ \\
\hline $4 \mathrm{CEI}(n=7)$ & $243 \pm 1$ & $43 \pm 1$ & $112 \pm 4$ & $0.70 \pm 0.13$ & $86 \pm 5$ & $0.30 \pm 0.02$ & $297 \pm 29$ & $50 \pm 2$ & $15 \pm 1$ \\
\hline C vs. MP & NS & NS & NS & NS & $P<0.001$ & NS & $P<0.05$ & $P<0.001$ & NS \\
\hline C vs. MP/CEI & NS & NS & $P<0.005$ & NS & NS & NS & $P<0.05$ & $P<0.001$ & NS \\
\hline C vs. CEI & NS & NS & $P<0.010$ & NS & NS & NS & NS & $P<0.001$ & NS \\
\hline MP vs. MP/CEI & NS & NS & $P<0.001$ & NS & NS & NS & NS & $P<0.001$ & NS \\
\hline
\end{tabular}

Abbreviations used in this table: $\pi_{A}$, Afferent arteriolar colloid osmotic pressure; $\pi_{E}$, efferent arteriolar colloid osmotic pressure; $C_{A}$, afferent arteriolar plasma protein concentration; $C_{E}$, efferent arteriolar plasma protein concentration; $P_{E}$, efferent arteriolar hydraulic pressure; $P_{T}$, proximal tubule hydraulic pressure; $R_{T}$, total arteriolar resistance $\left(R_{A}+R_{E}\right)$; and $S N F F$, single nephron filtration fraction.

ies and arterioles showed rare hypertrophic changes of their media with minimal hyaline deposition. Qualitatively similar changes were observed in MP-treated group 6 rats. In addition, microthrombosis was frequently observed in segmentally damaged glomeruli in group 6 (Fig. $3 b$ ). The incidence of lesions in MP-treated rats was twice that of the untreated group, involving $40.5 \pm 6 \%$ of glomeruli $(P<0.05$ vs. group 5) (Fig. 4). In contrast, animals treated with MP/CEI (group 7) or CEI alone (group 8) showed minimal or no structural alterations (Fig. 3 $c$ ), with segmental glomerular lesions limited on average to $4 \pm 1 \%$ and $1.3 \pm 0.5 \%$ of glomeruli, respectively (both $P<0.05$ vs. groups 5 and 6) (Fig. 4).

\section{Discussion}

The efficacy of glucocorticoids as antiinflammatory and immunomodulatory agents, and their success in treatment of glomerular diseases such as membranous glomerulonephritis (31) and minimal change disease (32), have generally over-

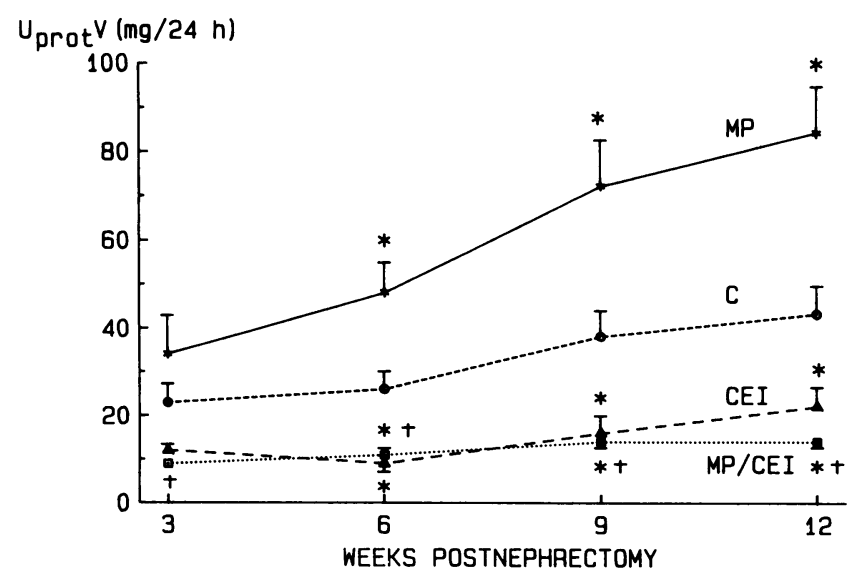

Figure 2. Urinary protein excretion rates $\left(U_{\text {prot }} \mathrm{V}\right)$. 24-h $U_{\text {prot }} \mathrm{V}$ in rats with five-sixths nephrectomy. Untreated rats (group 5, C) developed progressive proteinuria over the 12 -wk course. Treatment with MP (group 6, MP) accelerated proteinuria, while addition of CEI to MPtreated rats (group 7, MP/CEI), or CEI alone (group 8, CEI) significantly limited proteinuria. Values are means \pm SEM. $* P<0.05$ vs. $C$, $\dagger P<0.05$ vs. MP at the same time point. shadowed consideration of any theoretical adverse renal consequences. However, potential adverse consequences of glucocorticoid administration have been recognized for several decades. Glucocorticoids may aggravate proteinuria in children with chronic renal disease $(33,34)$, and repeated cortisone administration is associated with glomerular sclerosis in humans (35) and rabbits (36). Moreover, Steinberg (4) has recently pointed out that several older studies, including the classic reference on the subject (37), indicate that deterioration of renal function in patients with lupus nephritis treated with high dose steroids is comparable to (38) or even more rapid than (39) that seen in patients treated with lower doses. In patients receiving alternate day prednisone therapy for the nephrotic syndrome, it has been reported that proteinuria increases on the days therapy is given, in association with a numerical increment in GFR (40), compared with non-treatment days.

Controlled clinical trials in patients with lupus nephritis indicate that the probability of retaining renal function is greater in patients treated with cytotoxic drugs and low dose prednisone than in those treated with high dose prednisone alone (1-4). These observations confirm those previously documented in murine models of lupus nephritis $(4,39,41)$. Recent advances in the relatively safe administration of cytotoxic agents, together with the failure of steroids to effectively retard glomerular injury in experimental and clinical lupus nephritis, suggest that reevaluation of the role of glucocorticoid therapy in some glomerular diseases may be warranted.

The current findings suggest a hemodynamic basis for these older reports of steroid-induced disease acceleration. Glomerular capillary hyperfiltration, hyperperfusion, and particularly hypertension have been implicated as hemodynamic mediators of the structural injury that eventuates in rats with renal ablation (10-12, 16-19). In the current study, removal of five-sixths of the functioning renal mass again produced this hemodynamic pattern, with the expected development of proteinuria and glomerular sclerosis.

Administration of MP resulted in a slight elevation in systemic arterial pressure as compared to the untreated remnant kidney rats, although this increment did not achieve statistical significance. However, recent studies suggest that systemic hypertension may not cause significant glomerular injury unless 
Table I. (Continued)

\begin{tabular}{|c|c|c|c|c|c|c|c|c|c|}
\hline $\mathbf{P}_{\mathbf{E}}$ & $\overline{\Delta \mathbf{P}}$ & $\mathbf{k}_{\boldsymbol{r}}$ & $\mathrm{C}_{\mathrm{A}}$ & $C_{E}$ & $\mathbf{\Pi}_{\mathbf{A}}$ & $\boldsymbol{\Pi}_{\mathrm{E}}$ & $R_{A} \times 10^{10}$ & $R_{E} \times 10^{10}$ & $\mathrm{R}_{\mathrm{T}} \times 10^{10}$ \\
\hline$m m H g$ & $m m H g$ & $n l /(s \cdot m m H g)$ & $\mathrm{g} / 100 \mathrm{ml}$ & $\mathrm{g} / 100 \mathrm{ml}$ & $m m H g$ & $m m H g$ & $d y n \cdot s \cdot \mathrm{cm}^{-5}$ & $d y n \cdot s \cdot \mathrm{cm}^{-5}$ & $d y n \cdot s \cdot \mathrm{cm}^{-s}$ \\
\hline $20 \pm 0.3$ & $53 \pm 2$ & $0.043 \pm 0.004$ & $5.5 \pm 0.1$ & $8.2 \pm 0.3$ & $18 \pm 0.8$ & $33 \pm 2$ & $1.37 \pm 0.05$ & $1.29 \pm 0.14$ & $2.65 \pm 0.19$ \\
\hline $20 \pm 1$ & $70 \pm 2$ & $0.040 \pm 0.002$ & $5.9 \pm 0.1$ & $8.8 \pm 0.2$ & $20 \pm 0.5$ & $37 \pm 1$ & $0.86 \pm 0.10$ & $1.10 \pm 0.09$ & $1.96 \pm 0.14$ \\
\hline $17 \pm 1$ & $39 \pm 1$ & $0.100 \pm 0.011$ & $5.5 \pm 0.1$ & $7.7 \pm 0.2$ & $18 \pm 0.6$ & $30 \pm 1$ & $0.83 \pm 0.06$ & $0.63 \pm 0.08$ & $1.45 \pm 0.11$ \\
\hline $18 \pm 1$ & $35 \pm 2$ & $0.121 \pm 0.009$ & $5.4 \pm 0.1$ & $7.7 \pm 0.3$ & $17 \pm 0.5$ & $30 \pm 2$ & $1.00 \pm 0.11$ & $0.66 \pm 0.09$ & $1.66 \pm 0.16$ \\
\hline NS & $P<0.001$ & NS & NS & NS & NS & NS & $P<0.005$ & NS & $P<0.020$ \\
\hline NS & $P<0.001$ & $P<0.001$ & NS & NS & NS & NS & $P<0.005$ & $P<0.005$ & $P<0.001$ \\
\hline NS & $P<0.001$ & $P<0.001$ & NS & NS & NS & NS & $P<0.020$ & $P<0.005$ & $P<0.005$ \\
\hline NS & $P<0.001$ & $P<0.001$ & NS & $P<0.05$ & NS & $P<0.05$ & NS & $P<0.020$ & NS \\
\hline
\end{tabular}

the elevated pressures are transmitted to the glomerular capillary network. For example, in the spontaneously hypertensive rat, systemic hypertension does not aggravate the course of experimental glomerulonephritis (42-44). The spontaneously hypertensive rat is characterized by relatively high values for $\mathbf{R}_{A}$, which tend to prevent transmission of high systemic pressures into the glomerular capillary network and protect against glomerular capillary hypertension (45). When this protective afferent vasoconstriction is abolished by uninephrectomy, transmission of high systemic pressure into the glomerular capillary results in glomerular hypertension, which is associated with acceleration of the development of proteinuria and glomerular sclerosis (46). In the present micropuncture study, steroid administration augmented systemic arterial pressure by only $7 \%$, while values for $\overline{\mathbf{P}}_{\mathrm{GC}}$ increased by $23 \%$. Taken together, these findings suggest that increased glomerular
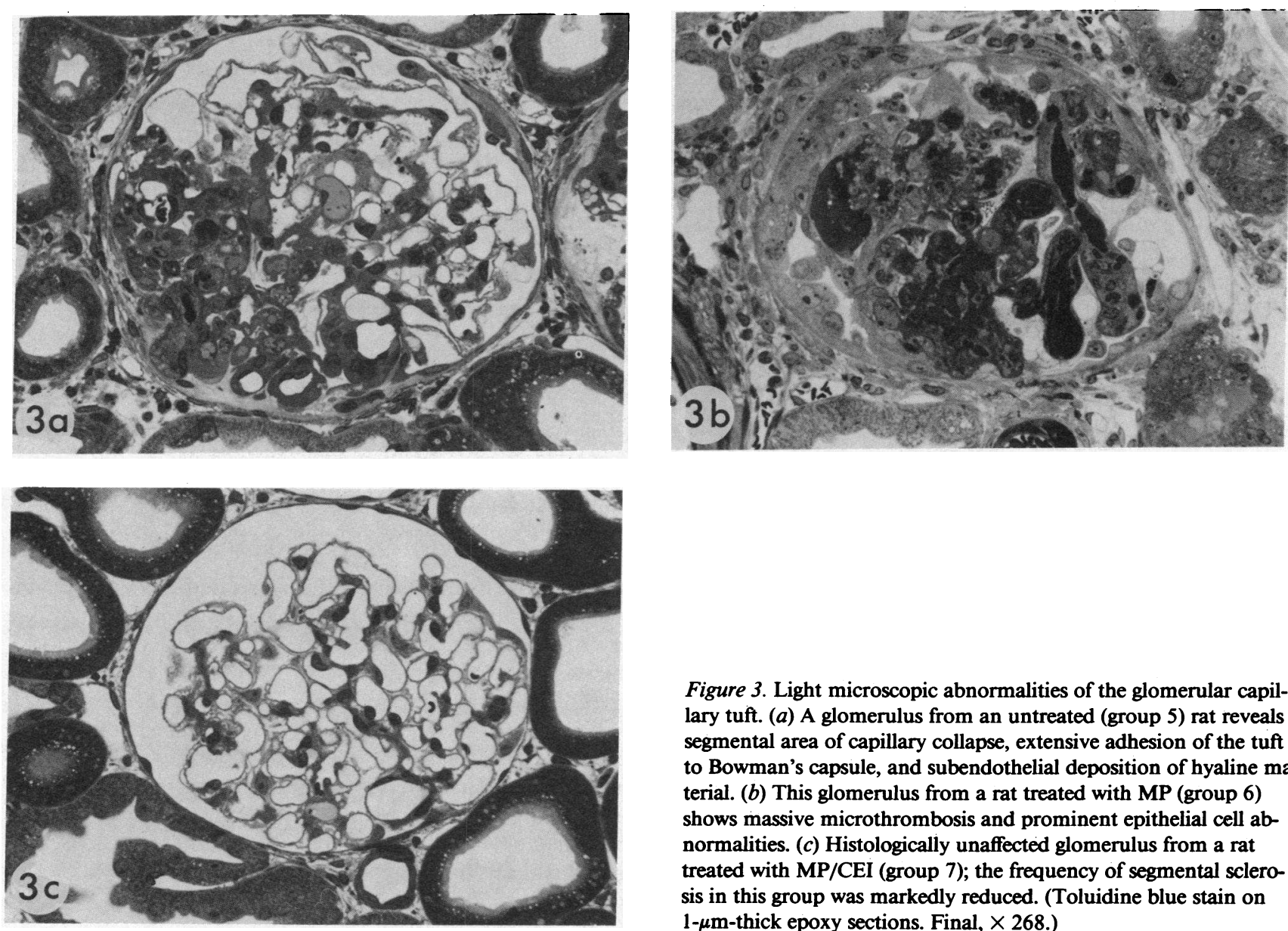

Figure 3. Light microscopic abnormalities of the glomerular capillary tuft. (a) A glomerulus from an untreated (group 5) rat reveals a segmental area of capillary collapse, extensive adhesion of the tuft to Bowman's capsule, and subendothelial deposition of hyaline material. (b) This glomerulus from a rat treated with MP (group 6) shows massive microthrombosis and prominent epithelial cell abnormalities. (c) Histologically unaffected glomerulus from a rat treated with MP/CEI (group 7); the frequency of segmental sclerosis in this group was markedly reduced. (Toluidine blue stain on $1-\mu$ m-thick epoxy sections. Final, $\times 268$.) 


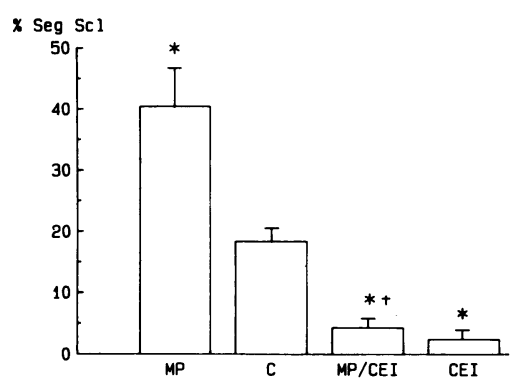

Figure 4. Percentage of glomeruli with focal and segmental glomerular sclerosis ( $\mathrm{Seg} \mathrm{Scl})$ at 12 wk. Treatment with MP (group 6, MP) resulted in doubling of the incidence of the lesions compared with untreated (group 5, C) rats. Addition of CEI to MP-treated rats (group

7, MP/CEI) or CEI alone (group 8, CEI) markedly reduced the incidence of segmental lesions. Values are means \pm SEM. $* P<0.05$ vs. $\mathrm{C}, \dagger P<0.05$ vs. MP.

rather than systemic hypertension contributed to the resultant glomerular injury, and that if this mild increase in systemic hypertension did contribute to the accelerated renal injury, that enhancement of glomerular hypertension may well have been the mechanism of injury.

In contrast to the minimal effects on systemic hypertension, steroid administration dramatically aggravated the adaptive glomerular hemodynamic response to reduction of renal mass. As compared with the untreated group 1 rats, MPtreated group 2 rats exhibited a further reduction in renal vascular resistance due to reductions in both $R_{A}$ and $R_{E}$ (Fig. 5). Because the decrease in $\mathbf{R}_{A}$ was proportionately greater than that in $R_{E}$, glomerular capillary hydraulic pressure increased even further. In the rats receiving MP/CEI (group 3) or CEI alone (group 4), both MP and reduction of systemic arterial pressure contributed to comparable reductions in values for $R_{A}$ (Fig. 5). However, in contrast to the findings in group 2, CEI therapy resulted in significantly reduced values for $R_{E}$ as well. In these latter groups, the concomitant reduction in $R_{E}$ served to offset the reduction in $\mathbf{R}_{\mathrm{A}}$, so that $\overline{\mathrm{P}}_{\mathrm{GC}}$ and therefore $\overline{\Delta \mathrm{P}}$ were reduced to near-normal levels.

In all four groups, intrarenal vasodilatation resulted in elevation of $\mathrm{Q}_{\mathrm{A}}$, which contributed to single nephron hyperfiltration. In the untreated group 1 and MP-treated group 2 rats, single nephron hyperfiltration was due to elevations in $Q_{A}$ and $\overline{\Delta P}$, while values for $K_{f}$ were depressed. In contrast, groups

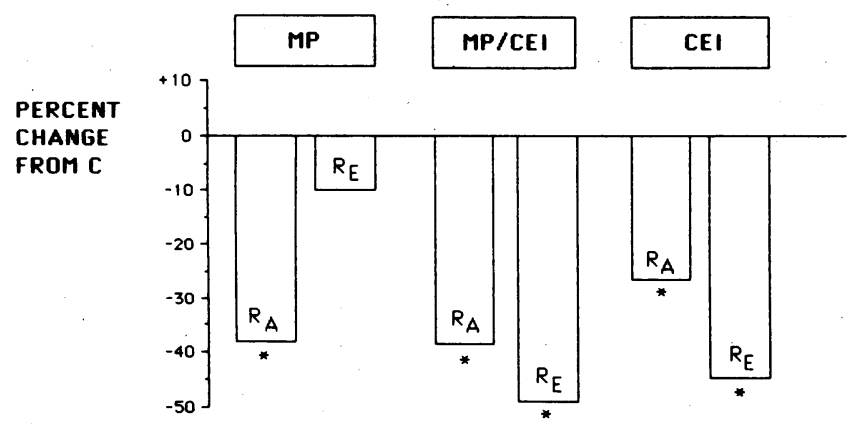

Figure 5. Percent change in renal resistances. As compared with values in untreated group 1 rats (C), treatment with MP in group 2 resulted in a greater reduction in $R_{A}$ than $R_{E}$, allowing an increase in glomerular hypertension. In groups 3 (MP/CEI) and 4 (CEI), comparable reductions in $R_{A}$ were offset by greater reductions in $R_{E}$, so that glomerular capillary pressure was markedly reduced. Values are mean percent change. $* P<0.05$ vs. $C$. receiving CEI alone or with MP exhibited single nephron hyperfiltration due to elevations in $Q_{A}$ and $K_{f}$.

In untreated group 1 rats, systemic and glomerular hypertension were associated with progressive glomerular structural injury, as manifested by increasing levels of proteinuria and glomerular sclerosis. Further enhancement of glomerular hyperfiltration, hyperperfusion, and hypertension with MP resulted in even higher rates of protein excretion, and doubling of the incidence of glomerular sclerotic lesions. Control of systemic and glomerular hypertension with CEI largely prevented these indices of glomerular structural injury. Therefore, in this experimental model of glucocorticoid-induced glomerular injury, control of glomerular hypertension is as effective as in streptozotocin diabetes (14) and renal ablation alone (11, 12 ), and is protective even in the absence of reductions in $Q_{A}$ and SNGFR.

Glomerular capillary hypertension in groups 1 and 2 was associated with subnormal values for $K_{f}$, whereas reduction of $\overline{\mathrm{P}}_{\mathrm{GC}}$ in the CEI-treated groups 3 and 4 was associated with preservation of normal values of $K_{f}$. In the untreated and MP-treated rats, it is unlikely that structural glomerular lesions figured prominently in the reduction in $\mathrm{K}_{f}$ seen only $2 \mathrm{wk}$ after ablation. It is quite possible that the low $\mathrm{K}_{\mathrm{f}}$ observed in untreated or MP-treated rats may have a functional basis, perhaps related to hormonal influences on mesangial cell function (47), since addition of CEI reversed the $K_{f}$ values toward the normal range. However, it is unlikely that changes in $K_{f}$ were causally related to structural modifications independent of changes in $\overline{\mathrm{P}}_{\mathrm{GC}}$, since the accelerated injury seen in MP-treated rats was associated with values for $K_{f}$ no different from those seen in untreated remnant kidney rats.

The present finding that glucocorticoids accelerate renal injury in a non-immunologic model of progressive renal disease is in accord with previous experimental studies in normal animals (36) as well as in mouse models of lupus nephritis (4, 39,41 ). Unfortunately, the absence of a rat model of lupus nephritis precludes a study of glomerular hemodynamics in this specific renal disease. However, inhibition of the reninangiotensin system with CEI (48) or with saralasin (49) may improve renal function (49) and improve outcome (48) in rats with puromycin nephrosis, a model of glomerular disease which, like human lupus nephritis (50), is characterized by absence of systemic hypertension and depression of single nephron filtration due to decreased values for $K_{f}$. Clearly, further studies of the potential hemodynamic consequences of steroid administration as well as concurrent inhibition of the renin-angiotensin system will be required to determine the applicability of the current findings to other models of glomerular injury.

The renal hemodynamic effects of alternative antiinflammatory agents such as cyclophosphamide and azathioprine have not been well characterized. More information is available regarding the immunosuppressive agent cyclosporin A, which produces renal vasoconstriction in humans (51) as well as in rats (52). Therapy with cyclosporin has been reported to reduce proteinuria in several experimental models of immune-mediated renal disease (53-55), as well as to retard the progression of non-immunologic glomerular injury in rats with renal ablation (56). It is presently unknown whether hemodynamic factors play a role in the protection afforded by these agents. 
In summary, we have shown that chronic administration of glucocorticoids to rats with extensive ablation of renal mass is associated with striking acceleration of glomerular injury due to further enhancement of the already elevated intraglomerular pressures and flows. Control of systemic and glomerular hypertension with concurrent administration of CEI results in protection against steroid-induced acceleration of progressive renal disease. Whether these undesirable hemodynamic effects of chronic steroid therapy occur in humans with renal disease or allografts, and whether they enhance the risk of progressive injury, remain to be determined.

\section{Acknowledgments}

Sheila Putnam provided expert secretarial assistance.

This study was supported by U. S. Public Health Service grant AM-35930 and by a grant from Ciba-Geigy Corp. Dr. Garcia is the recipient of a Research Fellowship Award from the National Kidney Foundation/Burroughs Wellcome Foundation; Dr. Anderson was the recipient of an Individual National Research Service Award of National Institutes of Health (S-F32-AM-07206).

\section{References}

1. Carette, S., J. H. Klippel, J. L. Decker, H. A. Austin, P. H. Plotz, A. D. Steinberg, and J. E. Balow. 1983. Controlled studies of oral immunosuppresive drugs in lupus nephritis: a long-term follow-up. Ann. Intern. Med. 99:1-8.

2. Balow, J. E., H. A. Austin, L. R. Muenz, K. M. Joyce, T. T. Antonovych, J. H. Klippel, A. D. Steinberg, P. H. Plotz, and J. L. Decker. 1984. Effect of treatment on the evolution of renal abnormalities in lupus nephritis. N. Engl. J. Med. 311:491-495.

3. Austin, H. A., J. H. Klippel, J. E. Balow, N. G. H. Le Riche, A. D. Steinberg, P. H. Plotz, and J. L. Decker. 1986. Therapy of lupus nephritis: Controlled trial of prednisone and cytotoxic drugs. N. Engl. J. Med. 314:614-619.

4. Steinberg, A. D. 1986. The treatment of lupus nephritis. Kidney Int. 30:769-787.

5. Baylis, C., and B. M. Brenner. 1978. Mechanism of the glucocorticoid-induced increase in glomerular filtration rate. Am. J. Physiol. 234:F166-G170.

6. Davis, J. O., and D. S. Howell. 1953. Comparative effects of ACTH, cortisone, and DOCA on renal function, electrolyte excretion and water exchange in normal dogs. Endocrinology. 52:245-255.

7. De Bermudez, L., and J. P. Hayslett. 1972. Effect of methylprednisolone on renal function and the zonal distribution of blood flow in the rat. Circ. Res. 31:44-52.

8. Levitt, M. F., and M. E. Bader. 1953. Effect of cortisone and ACTH on fluid and electrolyte distribution in man. Am. J. Med. 11:715-723.

9. Pechet, M. M., B. Bowers, and F. C. Bartter. 1959. Metabolic studies with a new series of 1,4-diene steroids. II. Effects in normal subjects of prednisone, prednisolone, and 9a-fluoroprednisolone. $J$. Clin. Invest. 38:691-701.

10. Hostetter, T. H., J. L. Olson, H. G. Rennke, M. A. Venkatachalam, and B. M. Brenner. 1981. Hyperfiltration in remnant nephrons: a potentially adverse response to renal ablation. Am. J. Physiol. 241:F85-F93.

11. Anderson, S., T. W. Meyer, H. G. Rennke, and B. M. Brenner. 1985. Control of glomerular hypertension limits glomerular injury in rats with reduced renal mass. J. Clin. Invest. 76:612-619.

12. Anderson, S., H. G. Rennke, and B. M. Brenner. 1986. Therapeutic advantage of converting enzyme inhibitors in arresting progressive renal disease associated with systemic hypertension in the rat. $J$. Clin. Invest. 77:1993-2000.

13. Zatz, R., T. W. Meyer, H. G. Rennke, and B. M. Brenner. 1985. Predominance of hemodynamic rather than metabolic factors in the pathogenesis of diabetic glomerulopathy. Proc. Natl. Acad. Sci. USA. 82:5963-5967.

14. Zatz, R., B. R. Dunn, T. W. Meyer, S. Anderson, H. G. Rennke, and B. M. Brenner. 1986. Prevention of diabetic glomerulopathy by pharmacological amelioration of glomerular capillary hypertension. J. Clin. Invest. 77:1925-1930.

15. Dworkin, L. D., T. H. Hostetter, H. G. Rennke, and B. M. Brenner. 1984. Hemodynamic basis for glomerular injury in rats with desoxycorticosterone-salt hypertension. J. Clin. Invest. 73:1448-1461.

16. Brenner, B. M., T. W. Meyer, and T. H. Hostetter. 1982. Dietary protein intake and the progressive nature of kidney disease: The role of hemodynamically mediated glomerular injury in the pathogenesis of progressive glomerular sclerosis in aging, renal ablation, and intrinsic renal disease. $N$. Engl. J. Med. 307:652-659.

17. Hostetter, T. H., T. W. Meyer, H. G. Rennke, and B. M. Brenner. 1986. Chronic effects of dietary protein in the rat with intact and reduced renal mass. Kidney Int. 30:509-517.

18. Olson, J. L., T. H. Hostetter, H. G. Rennke, B. M. Brenner, and M. A. Venkatachalam. 1982. Altered glomerular permselectivity and progressive sclerosis following extreme ablation of renal mass. Kidney Int. 22:112-126.

19. Meyer, T. W., S. Anderson, H. G. Rennke, and B. M. Brenner. 1987. Reversing glomerular hypertension stabilizes established glomerular injury. Kidney Int. 31:752-759.

20. Striker, G. E., R. B. Nagle, P. W. Kohnen, and E. A. Smuckler. 1969. Response to unilateral nephrectomy in old rats. Arch. Pathol. 87:439-442.

21. O'Donnell, M. P., B. L. Kasiske, F. X. Daniels, and W. F. Keane. 1986. Effect of nephron loss on glomerular hemodynamics and morphology in diabetic rats. Diabetes 35;1011-1015.

22. Banes, C. D., and L. G. Eltherington. 1973. Drug Dosage in Laboratory Animals. University of California Press, Berkeley. 278-279.

23. Pfeffer, J. M., M. A. Pfeffer, and E. D. Frohlich. 1971. Validity of an indirect tailcuff method for determining systolic arterial pressure in unanesthetized normotensive and spontaneously hypertensive rats. J. Lab. Clin. Med. 78:957-962.

24. Maddox, D. A., D. C. Price, and F. C. Rector, Jr. 1977. Effects of surgery on plasma volume and salt and water excretion in rats. $\mathrm{Am}$. J. Physiol. 233:F600-F606.

25. Deen, W. M., J. L. Troy, C. R. Robertson, and B. M. Brenner. 1973. Dynamics of glomerular ultrafiltration in the rat. IV. Determination of the ultrafiltration coefficient. J. Clin. Invest. 52:1500-1508.

26. Vurek, G. C., and S. E. Pegram. 1966. Fluorometric method for the determination of nanogram quantities of inulin. Anal. Biochem. 88:513-521.

27. Führ, J., J. Kaczmarczyk, and C. D. Krüttgen. 1955. Eine enfache colorimetrische Methode zur Inulinbestimmung fur Nierenclearance-Untersuchungen bei Stoffwechselgesunden und Diabetikern. Klin. Wochenschr. 33:729-730.

28. Viets, J. W., W. M. Deen, J. L. Troy, and B. M. Brenner. 1978. Determination of serum protein concentration in nanoliter blood samples using fluorescamine or o-phthalaldehyde. Anal. Biochem. 88:513-521.

29. Bradley, M., G. B. Schuman, and P. C. J. Ward. 1979. Examination of urine. Todd. Sanford. Davidsohn's Clinical Diagnosis and Management by Laboratory Methods. 16th ed. J. B. Henry, editor. W. B. Saunders Co., Philadelphia. 604-605.

30. Wallenstein, S., C. L. Zucker, and J. L. Fleiss. 1980. Some statistical methods useful in circulation research. Circ. Res. 47:1-9.

31. Collaborative study of the adult idiopathic nephrotic syndrome. 1979. A controlled study of short-term treatment in adults with membranous nephropathy. N. Engl. J. Med. 301:1301-1306.

32. A report of the international study of kidney disease in children. 1981. The primary nephrotic syndrome in children. Identification of patients with minimal change nephrotic syndrome from initial response to prednisone. J. Pediatr. 98:561-569. 
33. Heymann, W., and W. E. Grupe. 1969. Increase in proteinuria due to steroid medication in chronic renal disease. Pediatrics 74:356363.

34. Dowdle, E., and S. J. Saunders. 1957. The acute effect of hydrocortisone sodium succinate on the proteinuria of the nephrotic syndrome. S. Afr. J. Lab. Clin. Med. 3:39-47.

35. Oppenheimer, E. H., and J. R. Esterly. 1963. Glomerular lesions in the nephrotic syndrome and their relation to cortisone therapy. Bull. Johns Hopkins Hosp. 113:158-172.

36. Moran, T. J., S. M. Kurtz, and J. J. Vasquez. 1962. Diabetic and cortisone-induced renal lesions. A morphologic and immunohistochemical study. Lab. Invest. 11:240-254.

37. Pollak, V. E., C. L. Pirani, and R. M. Kark. 1961. Effect of large dose of prednisone on the renal lesions and life span of patients with lupus nephritis. J. Lab. Clin. Med. 57:495-511.

38. Kagan, L. J., and C. L. Christian. 1966. Clinicopathologic studies of SLE nephritis. Arthritis Rheum. 9:516. (Abstr.)

39. Steinberg, A. D., M. G. Gelfand, J. A. Hardin, and D. T. Lowenthal. 1975. Therapeutic studies in NZB/W mice. III. Relationship between renal status and efficacy of immunosuppressive drug therapy. Arthritis Rheum. 18:10-14.

40. Wetzels, J. F. M., P. G. G. Gerlag, H. E. Sluiter, A. J. Hoitsma, and R. A. P. Koene. 1986. Prednisone-induced fluctuations of proteinuria in patients with a nephrotic syndrome. Nephron. 44:344-350.

41. Steinberg, E. B., H. R. Smith, and A. D. Steinberg. 1983. Studies of cyclophosphamide therapy in murine lupus: Effect of combining multiple subsets into a single randomized study. Arthritis Rheum. 26:1293-1294.

42. Raij, L., S. Azar, and W. F. Keane. 1985. Role of hypertension in progressive glomerular immune injury. Hypertension 7:398-404.

43. Olson, J. L., S. K. Wilson, and R. H. Heptinstall. 1986. Relation of glomerular injury to preglomerular resistance in experimental hypertension. Kidney Int. 29:849-857.

44. Stein, H. D., R. B. Sterzel, J. D. Hunt, R. Pabst, and M. Kashgarian. 1986. No aggravation of the course of experimental glomerulonephritis in spontaneously hypertensive rats. Am. J. Pathol. 122:520-530.
45. Arendshorst, W. J., and W. H. Beierwaltes. 1979. Renal and nephron hemodynamics in spontaneousiy hypertensive rats. Am. J. Physiol. 236:F246-F251.

46. Dworkin, L. D., and H. D. Feiner. 1986. Glomerular injury in uninephrectomized spontaneously hypertensive rats. A consequence of glomerular capillary hypertension. J. Clin. Invest. 77:797-809.

47. Dworkin, L. D., I. Ichikawa, and B. M. Brenner. 1983. Hormonal modulation of glomerular function. Am. J. Physiol. F95-F104.

48. Anderson, S., J. R. Diamond, M. J. Karnovsky, and B. M. Brenner. 1987. Hemodynamic mechanisms of glomerular sclerosis after recovery from acute nephrotic syndrome. Proc. Xth Int. Cong. Nephrol., London. In press.

49. Ichikawa, I., H. G. Rennke, J. R. Hoyer, K. F. Badr, N. Schor, J. L. Troy, C. P. Lechene, and B. M. Brenner. 1983. Role for intrarenal mechanisms in the impaired salt excretion of experimental nephrotic syndrome. J. Clin. Invest. 71:91-103.

50. Myers, B. D., T. B. Okarma, S. Friedman, C. Bridges, J. Ross, S. Asseff, and W. M. Deen. 1982. Mechanisms of proteinuria in human glomerulonephritis. J. Clin. Invest. 670:732-746.

51. Curtis, J. J., E. Dubovsky, J. D. Whelchel, R. G. Luke, A. G. Diethelm, and P. Jones. 1986. Cyclosporin in therapeutic doses increases renal allograft vascular resistance. Lancet ii:477-479.

52. Murray, B. M., M. S. Paller, and T. F. Ferris. 1985. Effect of cyclosporine administration on renal hemodynamics in conscious rats. Kidney Int. 28:767-774.

53. Gunn, H. G., and B. Ryffel. 1986. Glomerulonephritis in NZB/W mice: therapeutic effect of cyclosporine. Clin. Nephrol. 25(Suppl. 1):S189-S192.

54. Thaiss, F., M. J. Mihatsch, S. Batsford, A. Vogt, and P. Schollmeyer. 1986. Effect of cyclosporine on in situ immune complex glomerulonephritis. Clin. Nephrol. 25(Suppl. 1):S181-S185.

55. Neild, G. H., K. Ivory, and D. G. Williams. 1986. Effect of cyclosporine on proteinuria in chronic serum sickness in rats. Clin. Nephrol. 25(Suppl. 1):S186-S188.

56. Brunner, F. P., M. Hermle, M. J. Mihatsch, and G. Thiel. 1986. Effect of cyclosporine in rats with reduced renal mass. Clin. Nephrol. 25(Suppl. 1):S148-S154. 